\title{
Effects of Pseudopotentials on the Phonon Dispersion of Graphene
}

\author{
K. N. Onaiwu ${ }^{1}$, J. O. A. Idiodi ${ }^{2}$ \\ ${ }^{I}$ Dept. of Physical and Earth Sciences, College of Natural and Applied Sciences, Crawford University, PMB \\ 2001, Igbesa, Ogun State, Nigeria. \\ ${ }^{2}$ Dept. of Physics, Faculty of Physical Sciences, University of Benin, Benin City, Edo State, Nigeria.
}

\begin{abstract}
The dependence of the various curves in the phonon dispersion of graphene on the particular pseudopotential used is analysed. By using three different pseudopotentials, we did a first principle calculation using the Quantum-ESPRESSO. It was found that the phonon frequencies of the various branches of graphene and some few layers graphene (FLGs) in addition to the crossings at some special symmetry points were dependent on the pseudopotential used.
\end{abstract}

Keyword: Graphene, phonon dispersion, pseudopotential, first principle, force constant

\section{Introduction}

The characteristic electronic properties of graphene (which is a single layer of graphite) in addition to the Dirac-like nature of the electrons near the Fermi level have made it a subject of public discuss in the scientific circle recently. The possibility of controlling the carrier density in the graphene sheet by simple application of a gate voltage [1] endows it with ballistic conduction at room temperature thus making it a potential candidate for both flexible transistors [2-5] and interconnects. In addition, it is emerging as an ultrasensitive probe for measuring strain $[6,7]$ just to mention but a few of such properties. Essentially, these properties are mediated by phonons. The phonon dispersion of graphite has not been fully resolved experimentally, this is not unrelated to the lack of large enough samples of crystalline quantity. It has been partly measured by inelastic neutron scattering (INS) [8], electron energy loss spectroscopy (EELS), and inelastic x-ray scattering (IXS) $[9,10]$.

Experimental data on the phonon dispersion of graphene are rare in the optical modes. Some of the few existing ones $[11,12]$ scatter widely and until the works by Ref. $[9,10]$ were missing completely for the $\mathrm{K}-\mathrm{M}$ direction. The dispersion of the transverse optical (TO) phonon mode is hardly known from the $\Gamma-$ point. The existing calculations [9, 13-17] leave much to be desired about the frequencies, slopes, and crossings of particular phonon branches. For example Ref. [18] calculated the phonon spectrum of graphene sheet, using a hybrid of the model proposed by Ref. [13], the results agreed partly with the IXS data of Ref. [10] but disagreed greatly at the K and M points for the TO and LO branches. Nevertheless, by extrapolating their IXS data, Ref. [10] obtained the TO frequency at the K-point to be $1265 \pm 10 \mathrm{~cm}^{-1}$ but alluded to the fact that their measured frequency was below $1300 \mathrm{~cm}^{-1 .}$. However, none of the references cited above came close to this value. In addition, most of the calculations [19-24] report that the transverse optical (TO) branch has a minimum at the K-point, while a local minimum is predicted by Refs. [25-26]. Also, the calculated frequencies at the K and M points differ by up to $300 \mathrm{~cm}^{-1}$, leading to differences in the slopes and even in the general shapes of the calculated phonon dispersions.

The contradiction in most existing calculations as spelt out above is the motivation behind this study. We calculate the phonon dispersion of graphene and some FLGs using the density functional theory (DFT) performed using Quantum-ESPRESSO (QE) codes [27] with the local density approximation (LDA). We used the Trouller Martin (TM) norm-conserving pseudopotentials and Rappe Rabe Kaxiras Joannopoulos ultra-soft (RRKJUS) pseudopotential, which is a Perdew-Zunger (LDA) exchange correlation functional. The wave functions were expanded using energy cut-off between 25-50 Ryd depending on the pseudopotential. We used Methfessel-Paxton smearing [28] in the calculations with an energy cut-off of 0.02 Ryd. A Monkhorst-Pack grid of $16 \times 16 \times 1$ was used for the Brillouin zone sampling. An in-plane lattice constant $a_{0}$ of 4.6595 a.u. (or $2.466 \AA$ ) and an interlayer distance of $10 a_{0}$ were used in the calculation. The results are fitted to the experimental data of Refs. $[9,10]$. We also compared the correlations and discrepancies between the experimental data and the calculated ones. 


\section{Method}

The phonon frequencies $\omega(\mathbf{q})$ are determined by solving the secular equation [29]:

$$
\left|\frac{1}{\sqrt{M_{s} M_{t}}} C_{s t}^{\alpha \beta}(\mathbf{q})-\omega^{2}(\mathbf{q})\right|=0
$$

$M_{s}$ and $M_{t}$ are the atomic masses of atoms $s$ and $t$ respectively and the dynamical matrix is defined as:

$$
C_{s t}^{\alpha \beta}(\mathbf{q})=\frac{\partial^{2} E}{\partial u_{s}^{* \alpha}(\mathbf{q}) \partial u_{t}^{\beta}(\mathbf{q})},
$$

where $u_{s}^{\alpha}$ denotes the displacement of atom $s$ in the direction $\alpha$. The second derivative of the energy in (2) is the change in the force $F_{t}^{\beta}(\mathbf{q})$ acting on atom $t$ in the direction $\beta$ due to a displacement of the atom $s$ in the direction $\alpha$ :

$$
C_{s t}^{\alpha \beta}(\mathbf{q})=\frac{\partial}{\partial u_{s}^{* \alpha}(\mathbf{q})} F_{t}^{\beta}(\mathbf{q})
$$

Because lattice distortion of a wave vector $\mathbf{q}$ does not induce a force response in the crystal at a wave vector $\mathbf{q}^{\prime} \neq \mathbf{q}$, interatomic force constants are most easily calculated in reciprocal space and by Fourier transformation, can be recovered back in direct space if needed. Coincidentally, the matrix of interatomic force constants is the sum of both the ionic and electronic contributions, i.e.:

where

$$
C_{s t}^{\alpha \beta}(\mathbf{q})={ }^{\mathrm{el}} C_{s t}^{\alpha \beta}(\mathbf{q})+{ }^{i o n} C_{s t}^{\alpha \beta}(\mathbf{q}),
$$

and

$$
{ }^{\mathrm{el}} C_{s t}^{\alpha \beta}(\mathbf{q})=\frac{1}{N_{c}}\left[\int\left(\frac{\partial n(\mathbf{r})}{\partial u_{s}^{\alpha}(\mathbf{q})}\right)^{*} \frac{\partial V_{\text {ion }}(\mathbf{r})}{\partial u_{t}^{\beta}(\mathbf{q})} d \mathbf{r}+\int n(\mathbf{r}) \frac{\partial^{2} V_{\text {ion }}(\mathbf{r})}{\partial u_{s}^{* \alpha}(\mathbf{q}) \partial u_{t}^{\beta}(\mathbf{q})} d \mathbf{r}\right],
$$

$$
V_{\text {ion }}(\mathbf{r})=\sum_{l s} v_{s}\left[\mathbf{r}-\mathbf{R}_{l}-\boldsymbol{\tau}_{s}-\mathbf{u}_{s}(l)\right]
$$

$N_{c}$ is the number of unit cells in the crystal $n(\mathbf{r})$ is the ground state electronic charge density, $\mathbf{R}_{l}$ the position of the $l$ th unit cell in the Bravais lattice, $\tau_{s}$ is the equilibrium position of the unit cell, $\mathbf{u}_{s}(l)$ is the deviation from equilibrium of the nuclear position and $v_{s}$ is the ionic (pseudo-) potential corresponding to the $s^{\text {th }}$ atomic species. For the explicit form of the ionic contribution to the matrix of interatomic force constants, ${ }^{i o n} C_{s t}^{\alpha \beta}(\mathbf{q})$ in (4), [ see the review by 29 , for details].

In the next section, we will show the dependence of the phonon frequencies on the pseudopotentials vis-a-vis the exchange-correlation functional implemented using the QE codes [27].

\section{Results}

Fig. 1 shows the phonon dispersion of a monolayer graphene calculated using the equilibrium lattice constant of 4.6595 a.u. obtained after the relaxation of the system. TM norm-conserving pseudopotentials were employed in the calculation and the Brillouin zone was sampled with a $16 \times 16 \times 1$ Monkhorst-Pack grid. Methefessel-Paxton smearing and an energy cut-off of $\sim 0.02$ Ryd. were used. Observe the crossings of the various branches at the $\mathrm{K}$ - and $\mathrm{M}$-points.

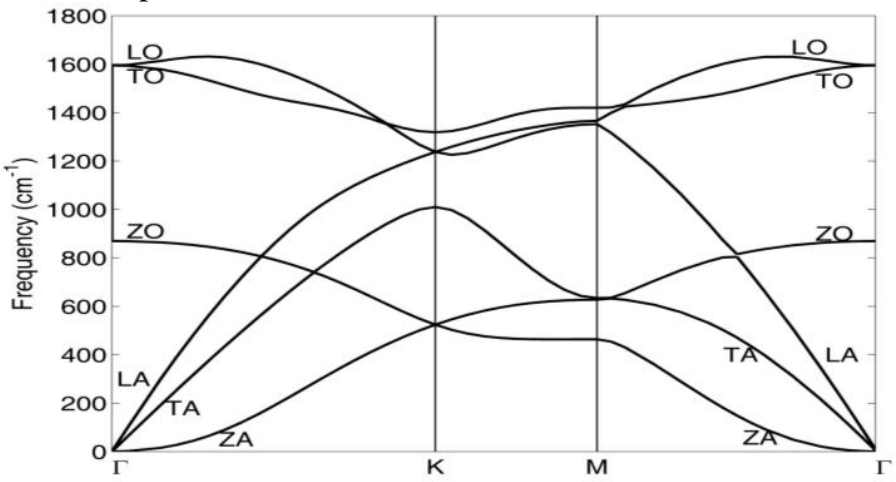

Figure. 1 Phonon dispersion of monolayer graphene. 
By replacing the pseudopotential with an ultrasoft pseudopotential (C.pz-rrkjus.UPF), the phonon dispersion shown in Fig. 2 was obtained. Observe, at the M point, the gap $\left(\sim 13 \mathrm{~cm}^{-1}\right)$ between the TO and LA branches in Fig. $2 \mathrm{~b}$ and that $\left(\sim 29.79 \mathrm{~cm}^{-1}\right)$ between the $\mathrm{ZO}$ and TA branches. This suggests that the type of pseudopotentials used could have significant effect(s) on the calculated frequencies both at the optical and acoustical regions. Observe the circle in (a) which is visibly absent from Fig. 1.

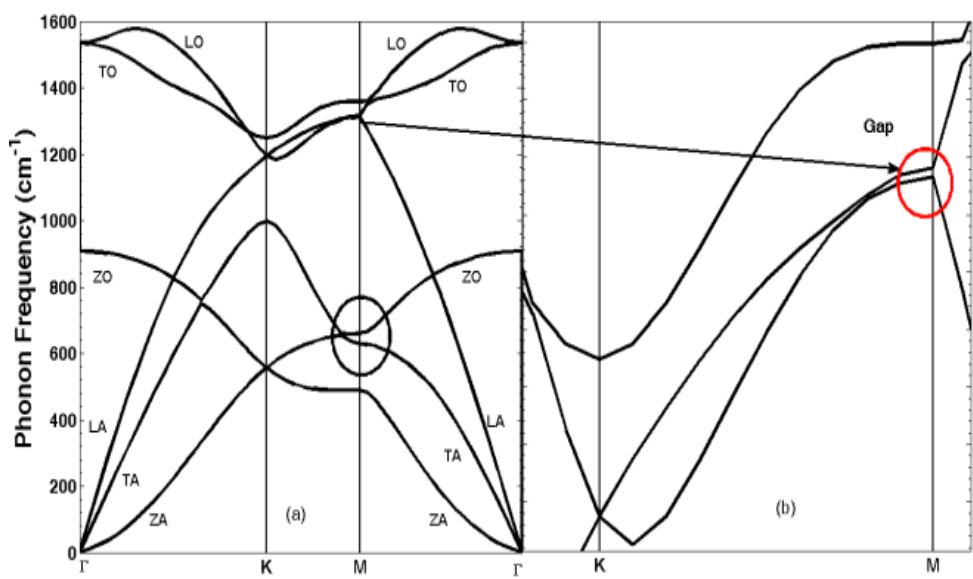

Figure 2: Phonon Dispersion of Graphene. (a) The phonon dispersion in the KM-direction and (b) Magnified view of the Optical Phonon in the KM direction. Observe the circled gaps in the two figures: in (a), ZO and TA cross each other before the special symmetry point $\mathrm{M}$ while the gap shown in the magnified view in (b) is not conspicuous in (a).

In Fig. 3, the calculated phonon dispersion is plotted along with the experimental data of Ref. [10], (triangles) and those of Ref. [9] (circles). Observe the close correlation between the experimental data and their calculated counterparts. On the contrary, the calculated data do not match excellently well with the INS data of Ref. [9] in the optical region for the choice of pseudopotential chosen for this calculation.

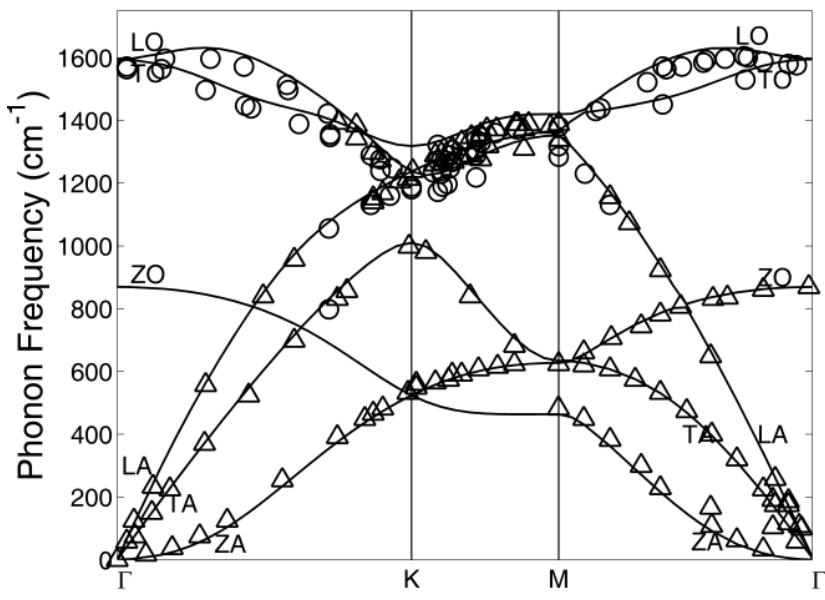

Figure 3: Calculated Phonon dispersion of graphene fitted to the IXS data of Ref. [10] (triangles) and the INS data of Ref. [9] (circles).

In the next section, we will explore the effects of different pseudopotentials to see how the slopes of the various branches are affected by the pseudopotential used.

\section{Effects Of Different Pseudopotentials}

In this section, the phonon dispersions of graphene using different pseudopotentials and by implication different exchange-correlation functionals are computed. Fig. 4 is obtained using an ultrasoft pseudopotential C_pbevan_bm_UPF (PS) which is a Perdew-Burke-Ernzerhof (PBE) exchange-correlation functional [30]. Observe that the phonon dispersion agrees largely with the experimental results of Refs. $[9,10]$ and that the gap between the LO and LA branches is more pronounced than it was in Figs. 1, 2 and 3. 


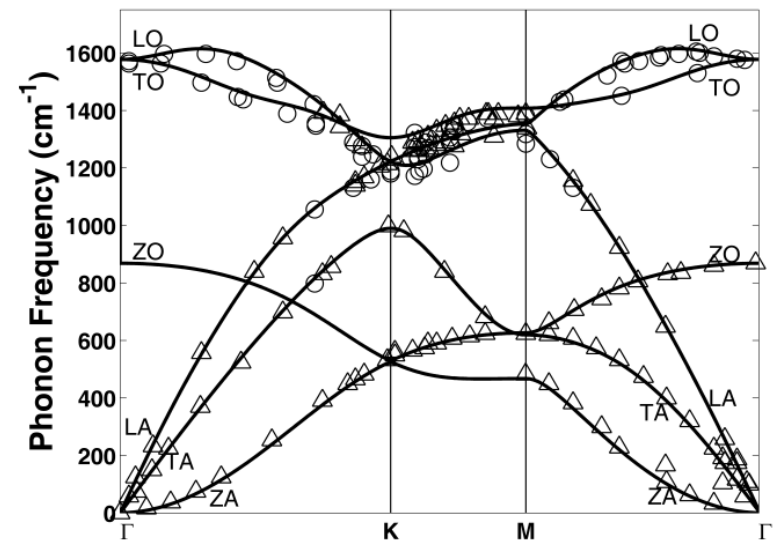

Figure 4: The phonon dispersion of graphene calculated using the pseudopotential C_pbe-van_bm_UPF. Circles are the INS data of Ref. [9] triangles are the IXS data of Ref. [10].

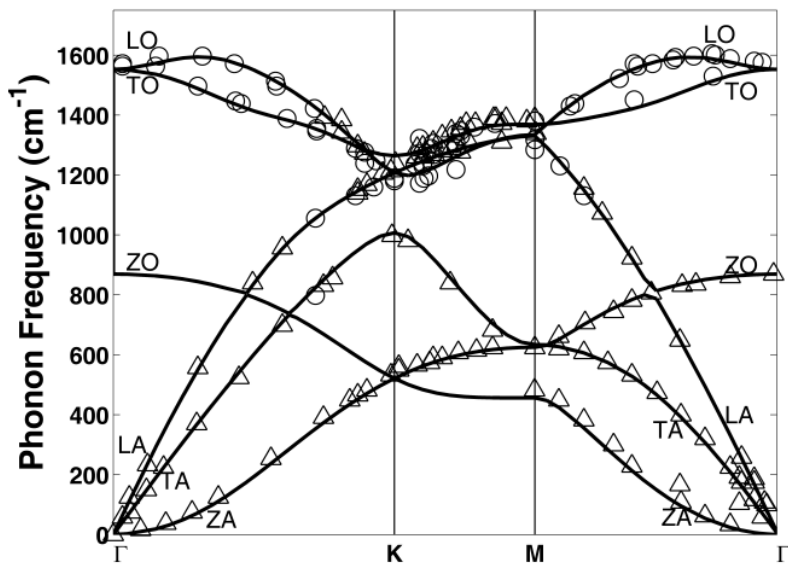

Figure 5: The phonon dispersion of graphene calculated using the pseudopotential C.pz-rrkjus.UPF. Circles are the INS data of Ref. [9] triangles are the IXS data of Ref. [10] and the solid line is our calculated points.

The result of the calculation done with an ultrasoft pseudopotential; the RRKJ pseudopotential C.pz-rrkjus.UPF (which is a Perdew-Zunger (LDA) exchange-correlation functional) [31] is presented in Fig. 6. Within the wave function cut-off explored in the calculation, it was observed that the result of the calculation does not match perfectly with the experimental data of Ref. 10 (triangles). This is observed especially along the ZO branch in the MГ- and KM-directions and led to the observed gap between the ZO and TA branches at the special k-point M. This gap is either missing or very narrow for the other pseudopotentials used thus far. Nevertheless, the agreement with the INS data of Ref. [9] especially in the optical region cannot be over-emphasized.

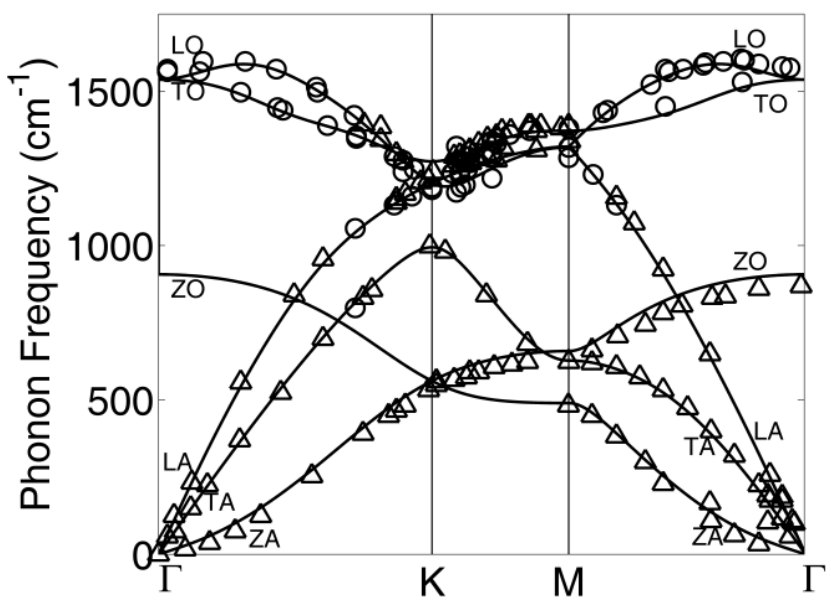

Figure 6: The phonon dispersion of graphene calculated using the pseudopotential C.pz-rrkjus.UPF. Circles are the INS data of Ref. [9], triangles are the IXS data of Ref. [10], and the solid line is our calculated points. 
The results of the calculations done, using the pseudopotentials C_pbe-van_bm_UPF and C.blyp_van.ak.UPF are shown in TABLE 1 for graphene and graphite along with some experimental data. The data presented for $\mathrm{AB}$ bilayer and $\mathrm{ABA}$ trilayer were obtained using C.blyp_van.ak.UPF. The first two entries under graphene are the present work; the first entries were obtained with the pseudopotential C_pbe-van_bm_UPF while in the second C.blyp_van.ak.UPF was used. The first entries under graphite are also the current work. Observe that the calculated TO frequencies $\left(1305 \mathrm{~cm}^{-1}, 1267 \mathrm{~cm}^{-1}\right)$ at the special symmetry point $\mathrm{K}$ are very close to the frequency, $1265 \mathrm{~cm}^{-1}$, that was obtained from the extrapolation of the IXS data of Maultzsch et al. [9]. However, the frequency obtained for the norm-conserving pseudopotential C blyp-mt UPF was much higher, $\sim 1327 \mathrm{~cm}^{-1}$.

Fig. 7 shows the phonon dispersion of some FLGs calculated using C.blyp van.ak.UPF. Although the phonon frequencies in (a) and (d) appear degenerate except at the frequencies below $200 \mathrm{~cm}^{-1}$ however, (b), (e) and (f) reveals that all the frequencies are non-degenerate.

TABLE 1: Phonon frequencies $\omega\left(\mathrm{cm}^{-1}\right)$ of Graphene and Graphite at some high symmetry points. The first two entries under Graphene are current work; the first entries were obtained with the pseudopotential C_pbevan_bm_UPF while in the second C.blyp_van.ak.UPF was used. All the other unreferenced data are the current work.

\begin{tabular}{|c|c|c|c|c|c|}
\hline \multicolumn{3}{|l|}{ k_point } & \multicolumn{3}{|c|}{ Frequency $\left(\mathrm{cm}^{-1}\right)$} \\
\hline & Graphene & $\mathbf{A B}$ & $\mathbf{A B A}$ & Graphite & ${ }_{\text {Graphite }(\operatorname{Expt})^{\mathrm{h}}}$ \\
\hline$\Gamma_{\mathrm{zO}}$ & $869,870,881^{\mathrm{a}}$ & 870 & 864 & $867,879^{\mathrm{b}}$ & $861^{b^{b}}$ \\
\hline$\Gamma_{\mathrm{LO}}$ & $1578,1552,1554^{\mathrm{a}}$ & 1578 & 1588 & $1575,1559^{\mathrm{b}}$ & $1590^{\mathrm{c}}, 1575^{\mathrm{d}}$ \\
\hline$\Gamma_{\mathrm{TO}}$ & $1578,1552,1554^{\mathrm{a}}$ & 1578 & & $1587,1567^{\mathrm{b}}$ & \\
\hline $\mathrm{M}_{\mathrm{ZA}}$ & $467,456,471^{\mathrm{a}}$ & 466 & 526 & $471,477^{\mathrm{b}}$ & $471^{\mathrm{f}}, 465^{\mathrm{c}}, 451^{\mathrm{g}}$ \\
\hline $\mathrm{M}_{\mathrm{TA}}$ & $620,625,626^{\mathrm{a}}$ & 621 & 620 & $621,634^{\mathrm{b}}$ & $630^{\mathrm{d}}$ \\
\hline $\mathrm{M}_{\mathrm{zO}}$ & $627,634,635^{\mathrm{a}}$ & 625 & 620 & 867,626 & $670^{\mathrm{c}}$ \\
\hline $\mathrm{M}_{\mathrm{LA}}$ & $1331,1330,1328^{\mathrm{a}}$ & 1336 & 1338 & $1335,1328^{\mathrm{b}}$ & $1290^{\mathrm{e}}$ \\
\hline $\mathrm{M}_{\mathrm{LO}}$ & $1353,1334,1340^{\mathrm{a}}$ & 1357 & 1365 & $1355,1340^{\mathrm{b}}$ & $1321^{\mathrm{e}}$ \\
\hline $\mathrm{M}_{\mathrm{TO}}$ & $1408,1368,1390^{\mathrm{a}}$ & 1411 & 1424 & $1410,1394^{\mathrm{b}}$ & $1388^{\mathrm{e}}, 1389^{\mathrm{c}}$ \\
\hline $\mathrm{K}_{\mathrm{ZA}}$ & $523,521,535^{\mathrm{a}}$ & 529 & 526 & $520,535^{\mathrm{b}}$ & $482^{\mathrm{g}}, 517 \mathrm{~g}, 530^{\mathrm{e}}$ \\
\hline $\mathrm{K}_{\mathrm{ZO}}$ & $533,521,535^{\mathrm{a}}$ & 531 & 527 & $527,539^{\mathrm{b}}$ & $588^{\mathrm{d}}, 627^{\mathrm{e}}$ \\
\hline $\mathrm{K}_{\mathrm{TA}}$ & $990,1007,997^{\mathrm{a}}$ & 993 & 991 & $993,998^{b}$ & \\
\hline $\mathrm{K}_{\mathrm{LA}}$ & $1223,1207,1213^{\mathrm{a}}$ & 1227 & 1232 & $1323,1216^{\mathrm{b}}$ & $1184^{\mathrm{e}}, 1202^{\mathrm{e}}$ \\
\hline $\mathrm{K}_{\mathrm{LO}}$ & $1221,1207,1213^{a}$ & 1227 & 1234 & $1225,1216^{\mathrm{b}}$ & $1184^{\mathrm{e}}, 1202^{\mathrm{e}}$ \\
\hline $\mathrm{K}_{\mathrm{TO}}$ & $1305,1264,1288^{\mathrm{a}}$ & 1311 & 1328 & $1323,1319^{\mathrm{b}}$ & $1313^{\mathrm{g}}, 1291^{\mathrm{e}}$ \\
\hline
\end{tabular}

${ }^{\mathrm{a}}$ Ref. [10], ${ }^{\mathrm{b}}$ Ref. [32], ${ }^{\mathrm{c}}$ Ref. [33], ${ }^{\mathrm{d}}$ Ref. [34], ${ }^{\mathrm{e}}$ Ref. [9], ${ }^{\mathrm{f}}$ Ref. [8], ${ }^{\mathrm{g}}$ Ref. [35], ${ }^{\mathrm{h}}$ As reported by Ref. [32], and the references therein.
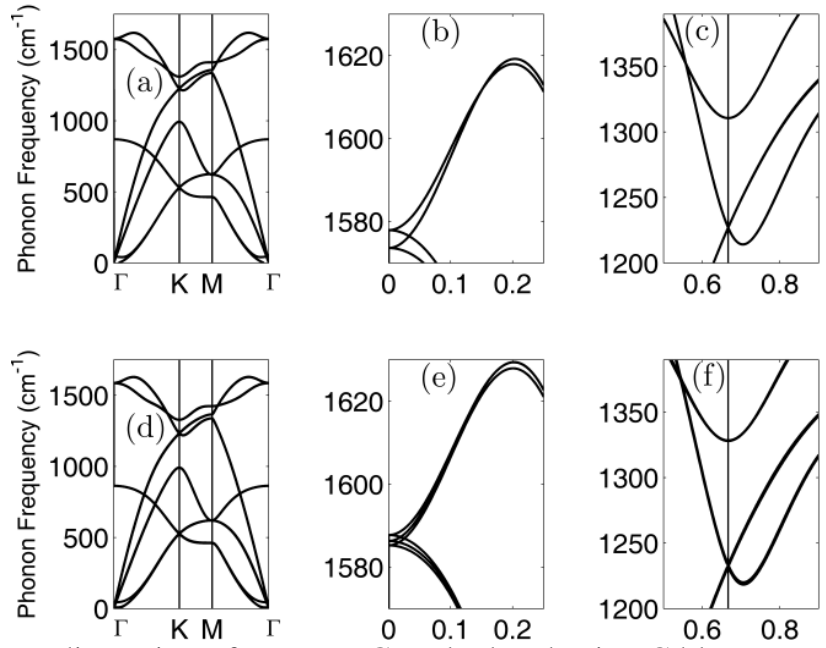

Figure. 7 below is the phonon dispersion of some FLGs calculated using C.blyp_van.ak.UPF. Though the phonon frequencies in (a) and (d) appear degenerate except at the frequencies below $200 \mathrm{~cm}^{-1}$ however, (b), (e) and (f) show that all the frequencies are actually non-degenerate.

\section{Conclusion}


The dependence of the various curves in the phonon dispersion of graphene on the particular pseudopotential used had been looked at in this work. We showed that the various branches and crossings of the phonon frequencies at the special k-points depend greatly on the pseudopotential, by implication the kinetic energy cutoff, used in the calculations. It was shown, see Fig. 5 for instance, that the TO branch has a minimum at the $\mathrm{K}$ symmetry point $\left(\sim 1264 \mathrm{~cm}^{-1}\right.$ for the pseudopotential C.blyp van.ak.UPF which is an ultrasoft pseudopotential and $\sim 1305 \mathrm{~cm}^{-1}$ for $\mathrm{C}$ pbe-van.ak.UPF which coincidentally is also an ultrasoft pseudopotential). We obtained the TO frequency at the neighbourhood of the $\Gamma$-point to be $1578 \mathrm{~cm}^{-1}$ and 1552 $\mathrm{cm}^{-1}$ respectively for the two pseudopotentials reported in our table as compared to the value reported by Ref. [9]. For completeness, we included the phonon dispersion of graphite, AB bilayer and ABA trilayer in TABLE 1.

\section{Acknowledgements}

We wish to express our thanks to the Centre for High Performance Computing (CHPC), South Africa, for allowing us use their resources. Our special thanks to N. Monama for his assistance and N. Omehe for the useful discussions we had.

\section{References}

[1]. K.S. Novoselov, A.K. Geim, S.V. Morozov, D. Jiang, M.I. Katsnelson, I.V. Grigorieva, S.V. Dubonos, A.A. Firsov, Electric Field Effect in Atomically Thin Carbon Films, Science 306 (2004) 666-669.

[2]. S. Cedric, A. Florence, L. Sylvie, T.S. Jung-Woo, C.H. Mark, D. Gilles, H. Henri, D. Vincent, Flexible Gigahertz Transistors Derived from Solution-Based Single-Layer Graphene, Nano Lett. 12 (2012) 1184.

[3]. F. Schwierz, Graphene Transistors, Nature Nanotech. 10 (2010) 487-496.

[4]. Y-M. Lin, et al., Operation of graphene transistors at gigahertz frequencies, Nano Lett. 9 (2009) 422-426.

[5]. F. Xia, D.B. Farmer, Y-M. Lin, P. Avouris, Graphene field-effect transistors with high on/off current ratio and large transport band gap at room temperature, Nano Lett. 10 (2010) 715-718.

[6]. T. Yu, N. Zhenhua, D. Chaoling, Y. Yumeng, W. Yingying, S. Zexiang, Raman Mapping Investigation of Graphene on Transparent Flexible Substrate: The Strain Effect. J. Phys. Chem. C 112 (2008) 12602.

[7]. T.M.G. Mohiuddin, A. Lombardo, R.R. Nair, A. Bonetti, G. Savini, R. Jalil, N. Bonini, D.M. Basko, C. Galiotis, N. Marzari, K.S. Novoselov, A.K. Geim, A.C. Ferrari, Uniaxial strain in graphene by Raman spectroscopy: G peak splitting, Grüneisen parameters, and sample orientation, Phys. Rev. B 79 (2009) 205433.

[8]. R. Nicklow, N. Wakabayashi, H.G. Smith, Lattice Dynamics of Pyrolytic Graphite, Phys. Rev. B 5 (1972) $4951-4962$.

[9]. J. Maultzsch, S. Reich, C. Thomsen, H. Requardt, P. Ordejón, Phonon Dispersion in Graphite, Phys. Rev. Lett. 92 (2004) 075501.

[10]. M. Mohr, J. Maultzsch, E. Dobardžić, S. Reich, I. Milošević, M. Damnjanović, et al., Phonon dispersion of graphite by inelastic xray scattering. Phys. Rev. B (2007) 035439.

[11]. S. Siebentritt, P. Roland, K. Rieder, Surface phonon dispersion in graphite and in a lanthanum graphite intercalation compound, Phys. Rev. B 55 (1997) 7927-7934.

[12]. J.L.Wilkes, J. Electron Spectrosc. Relat. Phenom. 44 (1987) 355.

[13]. T. Lenosky, X.T. Gonze, V. Elser, Energetics of negatively curved graphitic carbon. Nature 355 (1992) $333-335$.

[14]. J. de Launay, Solid State Phys. 3 (1957) 203.

[15]. L. Lang, S. Doyen-Lang, A. Charlier, M.F. Charlier, Dynamical study of graphite and graphite intercalation compounds. Phys. Rev. B 49 (1994) 5672-5681.

[16]. Y. Jia-An, W.Y. Ruan, M.Y. Chou, Phonon dispersions and vibrational properties of monolayer, bilayer, and trilayer graphene: Density-functional perturbation theory, Phys. Rev. B 77 (2008) 125401.

[17]. L.A. Falvosky, Phonon Dispersion in Graphene, J. Expt. and Theor. Phys. 105 (2007) 397-403.

[18]. S.K. Viola, D. K. Campbell, N.A.H. Castro, Lenosky's energy and the phonon dispersion of graphene, Phys. Rev. B 80 (2009) 035401.

[19]. C. Mapelli, C. Castiglioni, G. Zerbi, K. Müllen, Common force field for graphite and polycyclic aromatic hydrocarbons, Phys. Rev. B 60 (1999) 12710-12725.

[20]. O. Dubay, G. Kresse, Accurate Density Functional Calculations for the Phonon Dispersion Relations of Graphite Layer and Carbon Nanotubes, Phys. Rev. B 67 (2003) 035401.

[21]. P. Pavone, R. Bauer, K. Karch, O. Schütt, S. Vent, W. Windl, D. Strauch, S. Baroni, S. S. de Gironcoli, Physica B 219-220 (1996) 439-441.

[22]. P. Sánchez, A. Emilio, M.S. Jóse, R. Angel, O. Pablo, Ab-initio structural, elastic, and vibrational properties of carbon nanotubes, Phys. Rev. B 59 (1999) 12678-12688.

[23]. L.A. Falvosky, Symmetry constraints on phonon dispersion in graphene, Cond-mat/0802.0912v1 (2008).

[24]. L.J. Karssemeijer, F. Annalisa, Phonons of graphene and graphitic materials derived from the emperical potential LCBOPII , condmat/1010.5594v1 (2010).

[25]. R. Saito, A. Jorio, A.G.F. Souza, G. Dresselhaus, M.S. Dresselhaus, M.A. Pimenta, Probing Phonon Dispersion Relations of Graphite by Double Resonance Raman Scattering, Phys. Rev. Lett. 88 (2001) 027401.

[26]. R.A. Jishi, G. Dresselhaus, Lattice-dynamical model for graphite, Phys. Rev. B 26 (1982) 4514-4522.

[27]. P. Giannozzi, et al., The Quantum-ESPRESSO code, http://dx.doi.org/10.1088/0953-8984/21/39/395502 (2009).

[28]. M. Methfessel, A.T. Paxton, High-Precision Sampling for Brillouin-Zone Integration in Metals. Phys. Rev. B 40 (1989) 3616-3621.

[29]. S. Baroni, S. de Gironcoli, A. Dal Corso, Phonons and related crystal properties from density-functional perturbation theory, Rev. Mod. Phys. 73 (2001) 515-562.

[30]. J.P. Perdew, K. Burke, M. Ernzerhof, Generalized Gradient Approximation Made Simple, Phys. Rev. Lett. 77 (1996) $3865-3868$.

[31]. A.M. Rappe, K.M. Rabe, E. Kaxiras, J.D. Joannopoulos, Optimized Pseudopotentials, Phys. Rev. B. 41 (1990) 1227-1230.

[32]. M. Nicolas, M. Nicola, First-principles determination of the structural, vibrational and thermodynamic properties of diamond, graphite, and derivatives, Phys. Rev. B. 71 (2005) 205214.

[33]. C. Oshima, T. Aizawa, R. Souda, Y. Ishizawa, Y. Sumiyoshi, Ultra-thin epitaxial films of graphite and hexagonal boron nitride on solid surfaces, Solid State Commun. 65 (1988) 1601.

[34]. F. Tuinstra, J.L. Koenig, Raman Spectrum of Graphite, J. Chem. Phys. 53 (1970) 1126. 
[35]. H. Yanagisawa, T. Tanaka, Y. Ishida, M. Matsue, E. Rokuta, M. Otani, S. Oshima, Surf. Interface Anal. 37 (2005) 133. 